Published in final edited form as:

Exp Gerontol. 2010 April ; 45(4): 286-290. doi:10.1016/j.exger.2009.12.010.

\title{
Mechanisms of Hematopoietic Stem Cell Aging
}

\author{
Aysegul V Ergen ${ }^{1}$ and Margaret A Goodell ${ }^{1,2, *}$ \\ ${ }^{1}$ Program in Developmental Biology, Baylor College of Medicine, Texas 77030, USA \\ ${ }^{2}$ Department of Pediatrics, Baylor College of Medicine, Texas 77030, USA
}

\section{Abstract}

New blood cells are continually produced from the hematopoietic stem cells (HSCs) that reside in the bone marrow. Throughout the life-span of the organism, this stem cell reservoir sustains life. Although HSCs can persist in vivo longer than one life-span (Harrison, Astle et al. 1978), with aging, HSC regenerative potential diminishes and skewing from lymphopoiesis toward myelopoiesis occurs. The expansion in the HSC pool with aging provides sufficient, yet abnormal, blood production. Examination of gene expression changes in aged HSCs has provided a link between aging and genomic instability. Furthermore, studies on the effects of reactive oxygen species (ROS) on HSC aging has given more insight into the reasons for HSC failure. Understanding of the interactions between niche cells and HSCs and changes in them with aging, may give us insights into the lineage skewing phenotype observed in the aged, and also other immune dysfunctions.

\section{Introduction}

Tissue-specific stem cells, which have the ability to self-renew to generate more stem cells or to differentiate to give rise to other cell types, maintain tissue homeostasis throughout life. In tissues with a higher turnover rate such as skin and blood, most of the differentiated progeny are lost through injury or use, thus tissue-specific stem cells must sustain tissue homeostasis and must compensate for the loss to avoid tissue or organ failures, whereas tissues with a lower turnover rate such as brain, residing stem cells have a limited capacity to repair. However, with aging, tissue-homeostasis is not maintained properly; functional decline in tissues may be due to the diminished stem cell function. To what extent stem cell aging contributes to this functional decline in tissues is not definitely answered, yet. Furthermore, the effect of the stem cell aging may be different from one tissue to another depending on turnover rate of the tissue itself.

Blood is one of the tissues that has very high turn-over rate, nonetheless it is not protected from age-related insults. Hematopoietic system aging is exhibited by increased incidence of myeloid proliferative diseases like MDS (Corey, Minden et al. 2007)(Corey et al., 2007) and cancer and by a decline in the adaptive immune system (Dorshkind, Montecino-Rodriguez et al. 2009) in humans. Since hematopoietic stem cells (HSC) sustain the blood system throughout life, those age-related changes could be due to the functional decline in HSCs. Although HSCs can sustain blood production for multiple life-spans, as shown by serial transplantation in mice (Harrison, Astle et al. 1978), recent studies showed that phenotypically and functionally they undergo dramatic changes during aging. The most profound effect is seen in the adaptive immune system: there is a marked decline in 
lymphoid function in the elderly. Additionally, aging results in overproduction of myeloid cells which leads to a pro-inflammatory environment.

Microarray studies on HSCs have revealed that multiple genes change expression over time (Rossi, Bryder et al. 2005) (Chambers, Shaw et al. 2007). Although these studies used different isolation schemes for HSCs, both studies showed coordinated regulation of genes with aging, such as myeloid lineage specification genes (Rossi, Bryder et al. 2005) and chromatin remodeling genes (Chambers, Shaw et al. 2007). These results indicate that HSC senescence is not the product of a few programmed genes, but a large number of genetic changes that affect entire biological systems. Changes in gene expression of epigenetic regulators (Chambers, Shaw et al. 2007) also provides a link between aging and epigenetics dysregulation which leads to aberrant gene expression and might cause malignant transformation and cancer.

Stem cells have the potential to rejuvenate many somatic tissues, but may be unable to do so efficiently because they themselves are subject to aging. By delineating the mechanisms of aging in HSCs at the molecular level, and understanding how stem cells interact with the aging niche, we hope to gain insights that would enable us to enhance the regenerative properties of aged stem cells.

\section{Aging of the Hematopoietic System}

Both adaptive and innate immune systems work together to respond to pathogens throughout the lifetime of an organism. Age-related changes in the immune system are explained by immunosenescence in which there is an increased pathogen sensitivity because of loss of lymphocyte proliferation and decreased affinity of antibodies to antigens. CD8+ T cells undergo oligoclonal expansion with aging, they are skewed toward previously encountered antigens and they fill up the niches in the peripheral immune tissues (Dorshkind, MontecinoRodriguez et al. 2009). Thus, terminally differentiated $\mathrm{T}$ cells can not respond to newly encountered viruses and naïve $\mathrm{T}$ cells can not replace those due to the lack of space. Furthermore, the B cell compartment generates antibodies with less affinity and B cells undergo class-switch recombination less effectively (Dorshkind, Montecino-Rodriguez et al. 2009). The myeloid branch does not show the same limited expansion phenotype. In contrast, skewing toward myleopoiesis becomes more pronounced and this leads to generation of a pro-inflammatory environment described as "inflammaging" (Franceschi, Capri et al. 2007). Both of these concepts; immunoaging and inflammaging, reveal that immune system aging is a complex mechanism involving all the cells that make up the whole blood system repertoire, especially HSCs, since they are the foundation of the blood system.

\section{Functional and Phenotypical Changes in Aging HSCs}

The increase in the absolute number of HSCs with aging in C57Bl/6 mouse strain seems to be contradictory to the proposed models of aging (Morrison, Wandycz et al. 1996; de Haan, Nijhof et al. 1997; Sudo, Ema et al. 2000; Rossi, Bryder et al. 2005; Chambers, Shaw et al. 2007). It has been thought that loss of hemaostasis in tissues with aging is caused by the loss of stem cell reservoirs. However, when it is measured both by cell surface marker expression and Hoechst dye efflux properties, a 3 to 10 fold increase in absolute number is observed as the mouse ages (Morrison, Wandycz et al. 1996; Sudo, Ema et al. 2000; Kim, Moon et al. 2003). HSCs also become more homogeneous for cell surface expression of Sca-1 and c-Kit, two canonical stem cell markers.

Murine HSCs have the ability to reconstitute the blood upon transplantation which is the standard assay to test HSC function. In serial transplantation settings, it has been shown that 
aged HSCs contribute to blood production through successive recipients even better than the younger counterparts (Harrison, Astle et al. 1978; Harrison 1979). Although these data provide evidence that aged HSCs are functionally normal, other assays do not support this hypothesis. When HSCs from old mice were assayed for function, several deficiencies came to light, including altered homing and mobilization properties (Liang, Van Zant et al. 2005; Xing, Ryan et al. 2006) and diminished competitive repopulating ability (Sudo, Ema et al. 2000; Rossi, Bryder et al. 2005; Chambers, Shaw et al. 2007). In competitive transplantation experiments, HSCs are mixed with young 'competitor' bone marrow which is able to rescue the recipient animal post-irradiation, so HSCs are permitted to proliferate in the absence of the requirement for animal survival. In those competitive transplants (Kim, Moon et al. 2003; Rossi, Bryder et al. 2005; Chambers, Shaw et al. 2007), long-term HSC function declined with aging thus, there is a deficit in HSC output.

Sudo and colleagues found that a subset of aged HSC have an abnormal differentiation program although they reconstituted the blood long- term and could self-renew as demonstrated by re-transplantation into a second round of transplant recipients (Sudo, Ema et al. 2000). These aged HSCs had myeloid biased differentiation, and could not provide balanced lymphopoiesis. These studies demonstrated that although there is a functional decline in a per-cell manner, the increase in HSC number compensates for this, but repopulation is not balanced; instead, there is overproduction of myeloid lineages.

In recent studies, it has been shown that HSC compartment is heterogeneous with a number of clonal lineages which differ in self-renewal, differential potential and cell cycle kinetics (Sieburg, Cho et al. 2006). Thus with aging, the composition of HSC pool may change and certain clones may be enriched within this aged microenvironment. Seiburg-Muller proposed that myeloid biased HSC clone increases in number in the aged mice whereas lymphoid biased clone decreases in number which leads to overproduction of myeloid lineages in the elderly (Sieburg, Cho et al. 2006). Individual HSC from myeloid biased clones in both young and old donors have similar properties, thus the myelod biased differentiation of aged HSCs might be a reflection of the enrichment of myeloid biased clones in the HSC population. The myeloid biased clones may show a better fitness to the aging niche or systemic environment. An alternative model claims that HSC population as a whole changes with time in a non-cell autonomous manner due to the aging environment. In this model, myeloid biased differentiation may be seen as developmental stage specific during the life-time of the organism. Thus, further studies are required to determine the reasons for the lineage skewing phenotype observed in the aged HSC.

\section{Gene Expression Changes}

In order to investigate the underlying molecular mechanisms responsible for these ageassociated declines in individual HSC function, genome-wide expression profiling on HSCs purified from old and young mice were performed by two different groups. (Rossi, Bryder et al. 2005) (Chambers, Shaw et al. 2007). These two studies used different strategies for purification of HSCs, hence they found different sets of genes that change expression over time.

Rossi and colleagues found that 907 genes were age-regulated and most of the genes that are differentially expressed in young and old HSCs are lineage-specific genes. Genes that are involved in lymphoid specification found to be down-regulated with age whereas myeloid specifying genes were found to be up-regulated with age which is correlated with myeloid skewing phenotype of aging. They also observed increased expression of many protooncogenes with aging which have been implicated in myeloid leukemias. 
Our group also performed an expression profiling on HSCs purified from 2- 6- 12- and 21months old mice. From this microarray study, around 1500 genes were found to increase significantly in expression with age, while a similar number decreased. The biological processes enriched in up- or down-regulated gene lists were examined by gene ontology; it was found that stress response and inflammatory genes were up-regulated with age whereas chromatin remodeling and DNA repair genes were down-regulated (Chambers, Shaw et al. 2007). These data clearly demonstrate that HSCs are not protected from the ravages of age.

Our results also suggest that the pro-inflammatory microenvironment within the aging bone marrow stimulates genes in HSCs that otherwise would remain inactivated such as Pselectin (Sel-P). Sel-P is one of the most highly up-regulated genes, and encodes a cell adhesion protein specifically expressed by platelets. Several studies have identified a major role for Sel-P in the inflammatory response (Chen and Geng 2006).

Microarray analysis also identified up-regulation of several cell adhesion molecules. The ability of HSCs to engraft in bone marrow is greatly influenced by their homing properties. Since cell adhesion plays a critical role in the engraftment and mobilization of HSCs, aberrant up-regulation of adhesion genes might affect the ability of older HSCs to home to and seed the bone marrow, resulting in diminished long-term repopulation activity. One interesting finding is increased expression of stress-response or protein folding-genes which could be due to oxidative stress. This is consistent with mouse models. When regulation of intracellular ROS levels are disrupted, loss of HSC function has been observed (Ito, Hirao et al. 2004). While inflammatory and stress response genes are up-regulated with age, chromatin silencing, DNA repair and remodeling genes are down-regulated which may lead to increased genomic instability and alterations in the epigenome.

\section{Genomic Stability and Epigenetics}

Accumulated DNA damage and loss of DNA repair has been suggested to be one of the mechanisms underlying age-dependent stem cell decline. Mouse models which have mutations in DNA repair show deficits in stem cell function which supports this hypothesis. In a recent study, it has been shown that mice with a point mutation causing a hypomorphic allele in the DNA ligase IV (LigIV), essential for DNA double-strand break repair through non-homologous end-joining mechanism, causes several deficits in hematopoietic system. Mutant mice had reduced bone marrow cellularity and decreased self-renewal and agedependent decline in multipotent cells within the KSL population (Nijnik, Woodbine et al. 2007). In another study, HSCs from mice mutant for telomerase RNA, Ercc2 (involved in nucleotide excision repair) and Ku80 (involved in non-homologous end-joining) exhibited an increase in number with age whereas there was a decline in lymphoid progenitors (Rossi, Bryder et al. 2007). Mutant HSCs had also reduced long-term reconstitution in transplantation experiments which is pronounced with increased donor age. They also provided evidence for accumulation of $\gamma$-H2AX DNA repair foci in wild-type stem cells which indicates that DNA damage increases with age (Rossi, Bryder et al. 2007).

Those mouse models clearly demonstrate that functional DNA repair machinery is essential for HSC function and self-renewal which has been shown to diminish with aging. Downregulation of genes involved in DNA repair such as Xrcc1, Rad52, Xab2 in our microarray study could explain how loss of DNA repair leads to deficits in stem cell function (Chambers, Shaw et al. 2007). Decline in DNA repair itself with aging has not been shown experimentally but one might claim that loss of proper DNA repair with aging would cause accumulation of DNA lesions which affects the stem cell function.

We also found other genes which are down-regulated with age and shown to be important for epigenetic stability. One example is Lamin A, which is important for maintaining the 
stability of the nuclear lamina. Mutations in the human $L M N A$ gene cause at least 11 different heritable diseases, which are collectively termed laminopathies, ranging from muscular dystrophies to premature aging (Worman and Bonne 2007).

Another link for the role of epigenetic factors on HSC function comes from studies on chromatin remodeling genes like Ezh2 and Bmi1 both are members of the Polycomb family of transcriptional repressors. Ezh2 was shown to be abundantly expressed by HSCs and down-regulated upon differentiation and implicated in senescence of mouse embryonic fibroblasts. (Kamminga, Bystrykh et al. 2006). Overexpression of Ezh2 in HSCs preserved stem cell potential in serial transplantations, whereas normal HSCs were exhausted after serial transplantations. Bmil was also shown to be required for HSC self-renewal and maintenance. Deficiency in Bmi1 resulted in several abnormalities such as alterations in hematopoietic lineages, shortened life-span and failure to thrive (Park, Qian et al. 2003). Studies on Bmil has implicated its role in the repression of p16 $6^{\mathrm{INK} 4 \mathrm{a}}$ and $\mathrm{p} 19^{\mathrm{Arf}}$ genes which leads to impaired HSC self-renewal (Jacobs, Kieboom et al. 1999; Oguro, Iwama et al. 2006). Interestingly, suppression of $\mathrm{p} 16^{\mathrm{INK} 4 \mathrm{a}} / \mathrm{p} 19^{\text {Arf }}$ locus by Bmil requires presencence of Ezh2 locus (Bracken, Kleine-Kohlbrecher et al. 2007) .

Furthermore, physical mapping of those genes that changed over time identified gene clusters on the chromosomes that were coordinately up or down-regulated with age which supports the idea that cellular aging may not depend entirely on cell autonomous genetic programs, but may reflect the impact of epigenetic factors that have the capacity to regulate gene expression at a global level (Chambers, Shaw et al. 2007). The striking up-regulation of Igkappa gene expression in aging HSC supports this model, since Igkappa transcription is normally prevented in HSC at least in part by DNA methylation and chromatin modifications (Schlissel 2004).

Aberrant gene expression observed during aging could be due to the loss of DNA repair and epigenetic regulation which is triggered by genotoxic stress such as oxidative stress and also repeated cell divisons which in turn may lead to malignant transformations.

\section{How does ROS affect HSC aging?}

Regulation of intracellular ROS levels is important since overproduction of ROS results in oxidative stress which causes DNA damage. In a recent study, it has been shown that HSCs could be separated into two sub-types based on intracellular ROS levels as ROS ${ }^{\text {low }}$ HSCs and ROS ${ }^{\text {high }}$ HSCs (Jang and Sharkis 2007). Both populations expressed the same HSC cell surface markers $\left(\mathrm{CD} 34^{-}, \mathrm{Lin}^{-}, \mathrm{Sca}-1^{+}\right.$and, $\left.\mathrm{c}-\mathrm{Kit}^{+}\right)$. They reported the ROS ${ }^{\text {low }}$ population had better self-renewal ability than ROS high, which loses this ability gradually through serial transplantation. Interestingly, the differentiation potential of the ROS ${ }^{\text {high }}$ population was skewed toward myelopoiesis which was comparable to aged mice. Treatment of ROS ${ }^{\text {high }}$ population with an antioxidant such as a p38 inhibitor was able to restore their activity. The authors concluded that the ROS ${ }^{\text {low }}$ population has the properties of the quiescent HSCs with long-term-repopulating activity. These data suggest that intracellular ROS levels control homeostasis and functional properties of HSCs (Jang and Sharkis 2007).

In another study, Atm gene involved in DNA damage checkpoint and responsible for genomic stability, was shown to be essential for HSC self-renewal. Atm deficiency has resulted in bone marrow failure after 24 weeks of age in mice due to a functional decline in HSCs which is due to the elevated levels of ROS (Ito, Hirao et al. 2004). The up-regulation of ROS levels led to activation of p38 mitogen activated protein kinase (MAPK), which in turn caused up-regulation of the cyclin-dependent kinase (cdk) inhibitors $\mathrm{p} 16^{\text {Ink4a }}$ and p19Arf. Cdk inhibitors probably inhibit HSC division required for self-renewal. Thus, Atm is 
required for HSC self-renewal. These findings provided evidence that intrinsic HSC exhaustion due to defective cell-cycle checkpoint responses may lead to HSC decline.

Another line of evidence for the role of ROS and HSC self-renewal comes from studies on FoxO transcription factors which play an important role for oxidative stress response and HSC self-renewal. Mice with triple conditional deletions of Foxo1, Foxo3a, and Foxo4 genes, had a significant decrease in hematopoietic stem and progenitors (Tothova, Kollipara et al. 2007). Moreover, Foxo3a single knock-out mice had defective HSC self-renewal and decreased expression of $\mathrm{p} 27^{\mathrm{Kip} 1}$ and $\mathrm{p} 57^{\mathrm{Kip} 2}$ which indicates that Foxo3a deficiency causes down-regulation of several cdk inhibitors resulting in dysregulation of HSC quiescence (Miyamoto, Araki et al. 2007). There is also increased levels of ROS in Foxo3a deficient HSCs (Miyamoto, Araki et al. 2007). These studies illustrate the importance of Foxo genes in the maintenance of HSC quiescence.

Bmi1 which has been implicated in aging through $\mathrm{p} 16^{\mathrm{INK} 4 \mathrm{a}}$ and $\mathrm{p} 19^{\mathrm{Arf}}$ repression, has been shown to play a role in maintaining mitochondrial function and redox homeostasis. A new study has found that cells derived from Bmil deficient mice have impaired mitochondrial function, increased levels of intracellular ROS and activation of DNA damage response (DDR) pathway (Liu, Cao et al. 2009). They also claimed that either treatment with antioxidant scavenger $\mathrm{N}$-acetylcysteine or genetic disruption of Chk2 improves many phenotypic deficiencies seen in Bmil deficient mice such as reduction of intracellular ROS levels in thymocytes and increases in thymus size. All these studies suggest that intracellular ROS levels and the related signaling pathways could be a major piece in this puzzle regulating the long-term self-renewal as well as activation, proliferation, differentiation and aging of HSCs. Then, another piece would be extrinsic factors and surrounding microenvironment which could have a direct impact on ROS levels and signaling pathways regulating HSC homeostasis.

\section{What is happening in the old niche?}

HSCs have been shown to be along the endosteal surface of trabecular bone in close proximity to both bone-forming osteoblasts and the endothelial cells that line blood vessels (Calvi, Adams et al. 2003; Zhang, Niu et al. 2003; Kiel, Yilmaz et al. 2005). These specialized locations are described as niches. One could argue that HSC composition changes with age since they produce more myeloid cells, this could also be true for the niche cells. There could be loss or expansion of one particular niche which may lead to a more homogeneous population skewed towards myeloid differentiation.

In muscle stem cells, Conboy and collegues pinpointed the effect of aged environment on the stem cells regenerative potential. They found factors present in the young systemic environment could restore proliferative and regenerative capacity of aged satellite (muscle stem cells) cells (Conboy, Conboy et al. 2005). They also observed aged satellite cells gave rise to more fibrogenic lineage than the myogenic lineage which is mediated by the factors present in the systemic environment of aged mice (Brack, Conboy et al. 2007).

Studies on HSCs also supporting the hypothesis that aged niche might have a role in lineage skewing phenotype observed in old animals which is also true for the satellite cells. By transplanting old and young bone marrow cells into old and young mice in a competitive setting, homing efficiencies were reduced both in old donors and recipients (Liang, Van Zant et al. 2005). In the same study, it has been shown that both old donors and recipients had skewing of engraftment toward myeloid lineages. In another study, transplanting old HSCs into young mice with a competitive bone marrow resulted in reduction of peripheral B lymphocytes. Whereas by transplanting young HSCs into old mice, B cell production was 
affected only in the short term (Rossi, Bryder et al. 2005). But both studies found increased myelopoiesis when they used either old donors or recipients.

Evidence supporting importance of the osteoblasts for the HSC aging comes from recent genetic study on Atr gene, conditional deletion of Atr gene has resulted in reduction in the volume of trabecular bone, thought to be the osteoblastic niche, which has caused premature appearance of aging-related phenotypes such as hair graying, alopecia, kyphosis, involution of the thymus and osteoporosis. Mutant mice had a significant depletion of hematopoietic stem and progenitor populations (KSL and early T-cell progenitors) and the regenerative capacity of these cells decreased. (Ruzankina, Pinzon-Guzman et al. 2007)

A finely tuned relationship between HSCs and niche cells is required for homeostasis, the balance could be disrupted by niche cells or by niche factors. Yet, we still have little information about which cells support HSCs and which factors they secrete to maintain HSC function and number. Thus, further studies are required to pinpoint this relationship and how it changes during aging.

\section{Conclusions}

We have accumulated knowledge about how HSCs change with aging, but still we do not know the mechanisms of aging. Gene expression studies and also mouse models suggest that DNA repair pathways and maintenance of genomic stability could be one of the driving forces for aging in HSCs. Loss of epigenetic regulation might account for those aberrant gene expression seen during aging. But it does not explain all the aspects of aging. However, the lineage skewing phenotype observed during aging might be due to the extrinsic factors like niche factors. Increased ROS generation and up-regulation of inflammatory response genes during HSC aging appear to have a direct effect on this process. Therefore, future studies will elucidate the inter-play between these potential mechanisms. By better understanding $\mathrm{HSC}$ aging we may be able to ameliorate the loss of regenerative potential of aged HSCs.

\section{Acknowledgments}

We acknowledge the support from Ellison Foundation.

\section{References}

Brack AS, Conboy MJ, et al. Increased Wnt signaling during aging alters muscle stem cell fate and increases fibrosis. Science. 2007; 317(5839):807-10. [PubMed: 17690295]

Bracken AP, Kleine-Kohlbrecher D, et al. The Polycomb group proteins bind throughout the INK4AARF locus and are disassociated in senescent cells. Genes Dev. 2007; 21(5):525-30. [PubMed: 17344414]

Calvi LM, Adams GB, et al. Osteoblastic cells regulate the haematopoietic stem cell niche. Nature. 2003; 425(6960):841-6. [PubMed: 14574413]

Chambers SM, Shaw CA, et al. Aging hematopoietic stem cells decline in function and exhibit epigenetic dysregulation. PLoS Biol. 2007; 5(8):e201. [PubMed: 17676974]

Chen M, Geng JG. P-selectin mediates adhesion of leukocytes, platelets, and cancer cells in inflammation, thrombosis, and cancer growth and metastasis. Arch Immunol Ther Exp (Warsz). 2006; 54(2):75-84. [PubMed: 16648968]

Conboy IM, Conboy MJ, et al. Rejuvenation of aged progenitor cells by exposure to a young systemic environment. Nature. 2005; 433(7027):760-4. [PubMed: 15716955]

Corey SJ, Minden MD, et al. Myelodysplastic syndromes: the complexity of stem-cell diseases. Nat Rev Cancer. 2007; 7(2):118-29. [PubMed: 17251918] 
de Haan G, Nijhof W, et al. Mouse strain-dependent changes in frequency and proliferation of hematopoietic stem cells during aging: correlation between lifespan and cycling activity. Blood. 1997; 89(5):1543-50. [PubMed: 9057635]

Dorshkind K, Montecino-Rodriguez E, et al. The ageing immune system: is it ever too old to become young again? Nat Rev Immunol. 2009; 9(1):57-62. [PubMed: 19104499]

Franceschi C, Capri M, et al. Inflammaging and anti-inflammaging: a systemic perspective on aging and longevity emerged from studies in humans. Mech Ageing Dev. 2007; 128(1):92-105. [PubMed: 17116321]

Harrison DE. Mouse erythropoietic stem cell lines function normally 100 months: loss related to number of transplantations. Mech Ageing Dev. 1979; 9(5-6):427-33. [PubMed: 37377]

Harrison DE, Astle CM, et al. Loss of proliferative capacity in immunohemopoietic stem cells caused by serial transplantation rather than aging. J Exp Med. 1978; 147(5):1526-31. [PubMed: 25943]

Ito K, Hirao A, et al. Regulation of oxidative stress by ATM is required for self-renewal of haematopoietic stem cells. Nature. 2004; 431(7011):997-1002. [PubMed: 15496926]

Jacobs JJ, Kieboom K, et al. The oncogene and Polycomb-group gene bmi-1 regulates cell proliferation and senescence through the ink4a locus. Nature. 1999; 397(6715):164-8. [PubMed: 9923679]

Jang YY, Sharkis SJ. A low level of reactive oxygen species selects for primitive hematopoietic stem cells that may reside in the low-oxygenic niche. Blood. 2007; 110(8):3056-63. [PubMed: 17595331]

Kamminga LM, Bystrykh LV, et al. The Polycomb group gene Ezh2 prevents hematopoietic stem cell exhaustion. Blood. 2006; 107(5):2170-9. [PubMed: 16293602]

Kiel MJ, Yilmaz OH, et al. SLAM family receptors distinguish hematopoietic stem and progenitor cells and reveal endothelial niches for stem cells. Cell. 2005; 121(7):1109-21. [PubMed: 15989959]

Kim M, Moon HB, et al. Major age-related changes of mouse hematopoietic stem/progenitor cells. Ann N Y Acad Sci. 2003; 996:195-208. [PubMed: 12799297]

Liang Y, Van Zant G, et al. Effects of aging on the homing and engraftment of murine hematopoietic stem and progenitor cells. Blood. 2005; 106(4):1479-87. [PubMed: 15827136]

Liu J, Cao L, et al. Bmil regulates mitochondrial function and the DNA damage response pathway. Nature. 2009; 459(7245):387-92. [PubMed: 19404261]

Miyamoto K, Araki KY, et al. Foxo3a is essential for maintenance of the hematopoietic stem cell pool. Cell Stem Cell. 2007; 1(1):101-12. [PubMed: 18371339]

Morrison SJ, Wandycz AM, et al. The aging of hematopoietic stem cells. Nat Med. 1996; 2(9):1011-6. [PubMed: 8782459]

Nijnik A, Woodbine L, et al. DNA repair is limiting for haematopoietic stem cells during ageing. Nature. 2007; 447(7145):686-90. [PubMed: 17554302]

Oguro H, Iwama A, et al. Differential impact of Ink4a and Arf on hematopoietic stem cells and their bone marrow microenvironment in Bmil-deficient mice. J Exp Med. 2006; 203(10):2247-53. [PubMed: 16954369]

Park IK, Qian D, et al. Bmi-1 is required for maintenance of adult self-renewing haematopoietic stem cells. Nature. 2003; 423(6937):302-5. [PubMed: 12714971]

Rossi DJ, Bryder D, et al. Deficiencies in DNA damage repair limit the function of haematopoietic stem cells with age. Nature. 2007; 447(7145):725-9. [PubMed: 17554309]

Rossi DJ, Bryder D, et al. Cell intrinsic alterations underlie hematopoietic stem cell aging. Proc Natl Acad Sci U S A. 2005; 102(26):9194-9. [PubMed: 15967997]

Ruzankina Y, Pinzon-Guzman C, et al. Deletion of the developmentally essential gene ATR in adult mice leads to age-related phenotypes and stem cell loss. Cell Stem Cell. 2007; 1(1):113-26. [PubMed: 18371340]

Schlissel MS. Regulation of activation and recombination of the murine Igkappa locus. Immunol Rev. 2004; 200:215-23. [PubMed: 15242407]

Sieburg HB, Cho RH, et al. The hematopoietic stem compartment consists of a limited number of discrete stem cell subsets. Blood. 2006; 107(6):2311-6. [PubMed: 16291588] 
Sudo K, Ema H, et al. Age-associated characteristics of murine hematopoietic stem cells. J Exp Med. 2000; 192(9):1273-80. [PubMed: 11067876]

Tothova Z, Kollipara R, et al. FoxOs are critical mediators of hematopoietic stem cell resistance to physiologic oxidative stress. Cell. 2007; 128(2):325-39. [PubMed: 17254970]

Worman HJ, Bonne G. "Laminopathies": a wide spectrum of human diseases. Exp Cell Res. 2007; 313(10):2121-33. [PubMed: 17467691]

Xing Z, Ryan MA, et al. Increased hematopoietic stem cell mobilization in aged mice. Blood. 2006; 108(7):2190-7. [PubMed: 16741255]

Zhang J, Niu C, et al. Identification of the haematopoietic stem cell niche and control of the niche size. Nature. 2003; 425(6960):836-41. [PubMed: 14574412] 